\title{
The Björling problem for minimal surfaces in a Lorentzian three-dimensional Lie group
}

\author{
Adriana A. Cintra • Francesco Mercuri • Irene I. Onnis
}

Received: 14 May 2014 / Accepted: 24 September 2014 / Published online: 9 October 2014

(C) Fondazione Annali di Matematica Pura ed Applicata and Springer-Verlag Berlin Heidelberg 2014

\begin{abstract}
In this paper, we will show the existence and uniqueness of the solution of the Björling problem for minimal surfaces in a three-dimensional Lorentzian Lie group.
\end{abstract}

Keywords Minimal surfaces · Weierstrass representation · Björling problem · Lorentzian manifold

Mathematics Subject Classification $53 \mathrm{~A} 10 \cdot 53 \mathrm{C} 42 \cdot 53 \mathrm{C} 50$

\section{Introduction}

The Weierstrass representation formula for minimal surfaces in $\mathbb{R}^{3}$ has been a fundamental tool for producing examples and proving general properties of such surfaces, since the surfaces can be parametrized by holomorphic data. In [18], the authors describe a general Weierstrass representation formula for simply connected minimal surfaces in an arbitrary Riemannian manifold. The partial differential equations involved are, in general, too complicated to be solved explicitly. However, for particular ambient 3-manifolds, such as the Heisenberg group, the hyperbolic space and the product of the hyperbolic plane with $\mathbb{R}$, the equations are more manageable and the formula can be used to produce examples (see $[15,18])$.

Work partially supported by Capes (Brazil) and CNPq (Brazil).

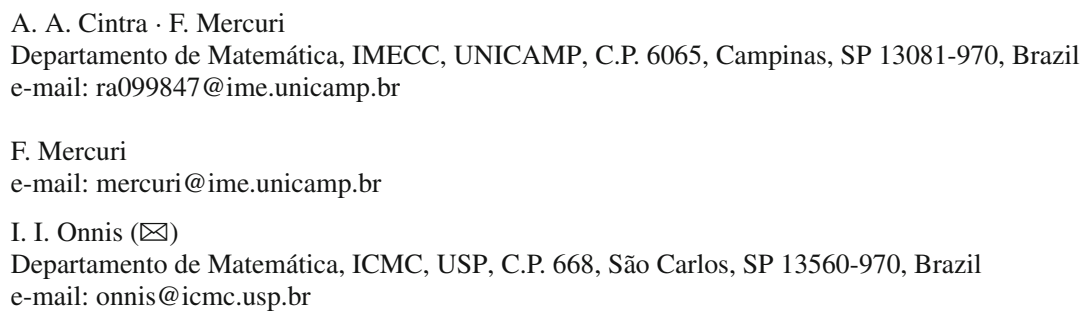


In the Lorentz-Minkowski space $\mathbb{L}^{3}$, i.e., the affine three space $\mathbb{R}^{3}$ endowed with the Lorentzian metric

$$
d s^{2}=d x_{1}^{2}+d x_{2}^{2}-d x_{3}^{2},
$$

a Weierstrass representation type theorem was proved by Kobayashi for spacelike minimal immersions (see [14]) and by Konderak for the case of timelike minimal surfaces (see [16]). Recently, these theorems were extended for minimal surfaces in Riemannian and Lorentzian three-dimensional manifolds by Lira et al. (see [17]).

The aim of this paper is to show how the Weierstrass representation formula can be used, if the ambient manifold is a three-dimensional Lorentzian Lie group, in order to prove existence and uniqueness of the solution of the Björling problem. We remember that the classical Björling problem, proposed by Björling in 1844 (see [5]), asks for the construction of a minimal surface in $\mathbb{R}^{3}$ containing a given analytic curve $\beta$ with a given analytic unit normal $V$ along it. The problem was solved by Schwarz in 1890 (see [22]) by means of an integral formula in terms of $\beta$ and $V$. Some extensions of this problem in others ambient spaces have been proposed and solved in $[3,4,8,9,19,20]$. Other geometric problems, inspired by the classical Björling problem, that can be formulated as a Cauchy problem for second-order partial differential equations may be consulted in [1,2,6,7,11-13].

The paper is organized as follows. In Sect. 2, we recall some basics facts of Lorentzian calculus, which plays the role of complex calculus in the classical case, for timelike minimal surfaces. Section 3 is devoted to present a Weierstrass- type representation for minimal surfaces in Lorentzian three-dimensional manifolds, following [17]. In Sect. 4, we state and solve the Björling problem for timelike and spacelike minimal surfaces in a Lorentzian three-dimensional Lie group. For timelike minimal surfaces, this is done by considering two different cases: When $\beta$ is a timelike curve, we will call the corresponding problem the timelike Björling problem, and when $\beta$ is a spacelike curve, we will have the spacelike Björling problem.

In Sects. 5, 6 and 7, we present some examples of minimal surfaces constructed via Björling problem for the case in which the ambient manifold is the Heisenberg group $\mathbb{H}_{3}$, the de Sitter space $\mathbb{S}_{1}^{3}$ and the space $\mathbb{H}^{2} \times \mathbb{R}$, equipped with left-invariant Lorentzian metrics.

\section{The algebra $\mathbb{L}$ of paracomplex numbers}

In [16], the author uses paracomplex analysis to deduce a Weierstrass representation formula for timelike minimal surfaces in $\mathbb{L}^{3}$. We recall that the algebra of paracomplex (or Lorentz) numbers is the algebra

$$
\mathbb{L}=\{a+\tau b \mid a, b \in \mathbb{R}\},
$$

where $\tau$ is an imaginary unit with $\tau^{2}=1$. The two internal operations are the obvious ones. We define the conjugation in $\mathbb{L}$ as $\overline{a+\tau b}:=a-\tau b$ and the $\mathbb{L}$-norm of $z=a+\tau b \in \mathbb{L}$ is defined by

$$
\| z||=|z \bar{z}|^{\frac{1}{2}}=\left|a^{2}-b^{2}\right|^{\frac{1}{2}} .
$$

The algebra $\mathbb{L}$ admits the set consisting of zero divisors $K=\{a \pm \tau a \in \mathbb{L}: a \neq 0\}$. If $z \notin K \cup\{0\}$, then $z$ is invertible with inverse $z^{-1}=\bar{z} /(z \bar{z})$.

We have that $\mathbb{L}$ is isomorphic to the algebra $\mathbb{R} \oplus \mathbb{R}$ via the map:

$$
\rho(a+\tau b)=(a+b, a-b) .
$$

The set $\mathbb{L}$ has a natural topology as a two-dimensional real vector space. 
Definition 1 Let $\Omega \subseteq \mathbb{L}$ be an open set and $z_{0} \in \Omega$. The $\mathbb{L}$-derivative of a function $f$ : $\Omega \rightarrow \mathbb{L}$ at $z_{0}$ is defined by

$$
f^{\prime}\left(z_{0}\right):=\lim _{\substack{z \rightarrow z_{0} \\ z-z_{0} \in \mathbb{L} \backslash K \cup\{0\}}} \frac{f(z)-f\left(z_{0}\right)}{z-z_{0}},
$$

if the limit exists. If $f^{\prime}\left(z_{0}\right)$ exists, we will say that $f$ is $\mathbb{L}$-differentiable at $z_{0}$.

Remark 1 The condition of $\mathbb{L}$-differentiability is much less restrictive that the usual complex differentiability. For example, $\mathbb{L}$-differentiability at $z_{0}$ does not imply continuity at $z_{0}$. However, $\mathbb{L}$-differentiability in an open set $\Omega \subset \mathbb{L}$ implies usual differentiability in $\Omega$.

Introducing the paracomplex operators:

$$
\frac{\partial}{\partial z}=\frac{1}{2}\left(\frac{\partial}{\partial u}+\tau \frac{\partial}{\partial v}\right), \quad \frac{\partial}{\partial \bar{z}}=\frac{1}{2}\left(\frac{\partial}{\partial u}-\tau \frac{\partial}{\partial v}\right),
$$

where $z=u+\tau v$, we have that a differentiable function $f: \Omega \rightarrow \mathbb{L}$ is $\mathbb{L}$-differentiable if and only if

$$
\frac{\partial f}{\partial \bar{z}}=0
$$

We observe that, writing $f(u, v)=a(u, v)+\tau b(u, v), u+\tau v \in \Omega$, the condition (2) is equivalent to the para-Cauchy-Riemann equations:

$$
\left\{\begin{array}{l}
\frac{\partial a}{\partial u}=\frac{\partial b}{\partial v} \\
\frac{\partial a}{\partial v}=\frac{\partial b}{\partial u}
\end{array}\right.
$$

whose integrability conditions are given by the wave equations

$$
a_{u u}-a_{v v}=0=b_{u u}-b_{v v} .
$$

\section{The Weierstrass representation formula in a Lorentzian 3-manifold}

We will denote by $\mathbb{K}$ either the complex numbers $\mathbb{C}$ or the paracomplex numbers $\mathbb{L}$, and by $\Omega \subset \mathbb{K}$ an open set. Let $(M, g)$ be a Lorentzian 3-manifold and $f: \Omega \subset \mathbb{K} \rightarrow M$ a smooth conformal immersion. We endow $\Omega$ with the induced metric that makes $f$ an isometric immersion. We will say that $f$ is spacelike if the induced metric on $\Omega$, via $f$, is a Riemannian metric, and that $f$ is timelike if the induced metric is a Lorentzian metric.

We observe that in the Lorentzian case, we can endow $\Omega$ with paracomplex isothermic coordinates and, as in the Riemannian case, they are locally described by paracomplex isothermic charts with conformal changes of coordinates. We will denote by $z=u+i v$ (respectively, $z=u+\tau v$ ) a complex (respectively, paracomplex) isothermal coordinate in $\Omega$.

The metric $g$ may be extended to $\mathbb{E}=f^{*} T M \otimes \mathbb{K}$ as:

- a (para)complex bilinear form (., .) : $\mathbb{E} \times \mathbb{E} \rightarrow \mathbb{K}$;

- a (para)Hermitian metric $\langle\langle.,\rangle\rangle:. \mathbb{E} \times \mathbb{E} \rightarrow \mathbb{K}$;

and the two extensions are related by:

$$
\langle\langle V, W\rangle\rangle=(V, \bar{W}) .
$$


Theorem 1 (Weierstrass Representation ([17])) Let $f: \Omega \subset \mathbb{K} \rightarrow M^{3}$ be a conformal minimal spacelike (or timelike) immersion and $g=\left(g_{i j}\right)$ be the induced metric. Define the (para)complex tangent vector $\phi \in \Gamma\left(f^{*} T M \otimes \mathbb{K}\right)$ by

$$
\phi(z)=\left.\frac{\partial f}{\partial z}\right|_{f(z)}=\sum_{i} \phi_{i} \frac{\partial}{\partial x_{i}} .
$$

Then, $\phi_{j}, j=1,2,3$ satisfy the following conditions:

(i) $\langle\langle\phi, \phi\rangle\rangle \neq 0$,

(ii) $(\phi, \phi)=0$,

(iii) $\frac{\partial \phi_{k}}{\partial \bar{z}}+\sum_{i, j=1}^{3} \Gamma_{i j}^{k} \bar{\phi}_{i} \phi_{j}=0$,

where $\left\{\Gamma_{i j}^{k}\right\}$ are the Christoffel symbols of $M$. Conversely, if $\Omega \subset \mathbb{K}$ is a simply connected domain and $\phi_{j}: \Omega \rightarrow \mathbb{K}, j=1,2,3$ are (para) complex functions satisfying the conditions above, then the map

$$
f: \Omega \rightarrow M, \quad f_{j}(z)=2 \mathcal{R} e \int_{z_{0}}^{z} \phi_{j} d z,
$$

is a well-defined conformal spacelike (or timelike) minimal immersion (here, $z_{0}$ is an arbitrary fixed point of $\Omega$, and the integral is along any curve joining $z_{0}$ to $z$ ).

Remark 2 The equation (iii) in Theorem 1 is a system of partial differential equations (really an integral differential equations, since the $\Gamma$ 's must be computed along a solution). Hence, in general, it is quite hard to find explicit solutions. However, for certain ambient spaces, these equations become a system of partial differential equations with constant coefficients (see Remark 3).

\subsection{The case of Lorentzian Lie groups}

Let $M$ be a three-dimensional Lie group endowed with a left-invariant Lorentzian metric $g$ and $\left\{E_{1}, E_{2}, E_{3}\right\}$ a left-invariant orthonormal frame field, with $E_{1}, E_{2}$ spacelike and $E_{3}$ timelike. For tangent vectors $W=\sum_{i=1}^{3} w_{i} E_{i}$ e $Y=\sum_{i=1}^{3} y_{i} E_{i}$, the Lorentzian cross product $Y \times W$ is given by:

$$
Y \times W=\left(y_{2} w_{3}-w_{2} y_{3}\right) E_{1}+\left(y_{3} w_{1}-w_{3} y_{1}\right) E_{2}+\left(y_{2} w_{1}-w_{2} y_{1}\right) E_{3} .
$$

It is easy to check that $Y \times W=-W \times Y$ and, also,

$$
\begin{aligned}
& g(U \times Y, W \times V)=g(U, V) g(Y, W)-g(U, W) g(Y, V), \\
& (U \times Y) \times W=g(Y, W) U-g(U, W) Y .
\end{aligned}
$$

Let $f: \Omega \subset \mathbb{K} \rightarrow M$ be a conformal minimal spacelike (or timelike) immersion, where $\Omega \subset \mathbb{K}$ is an open set. Fixed an isothermal parameter $z \in \Omega$, we can write the (para)complex tangent vector $\phi=\frac{\partial f}{\partial z}$ along $f$ both in terms of local coordinates $\left\{x_{1}, x_{2}, x_{3}\right\}$ in $M$ and, also, using the left-invariant frame field. Hence, one has

$$
\phi=\sum_{a=1}^{3} \phi_{a} \frac{\partial}{\partial x_{a}}=\sum_{a=1}^{3} \psi_{a} E_{a},
$$


where the functions $\phi_{a}$ and $\psi_{b}$, with $a, b=1,2,3$, are related by

$$
\phi_{a}=\sum_{b=1}^{3} A_{a b} \psi_{b}, \quad a=1,2,3,
$$

where $A: \Omega \rightarrow G L(3, \mathbb{R})$ is a smooth map. In terms of the components $\psi_{a}, a=1,2,3$, the equation (iii) in Theorem 1 may be written as

$$
\frac{\partial \psi_{c}}{\partial \bar{z}}+\frac{1}{2} \sum_{a, b=1}^{3} L_{a b}^{c} \bar{\psi}_{a} \psi_{b}=0, \quad c=1,2,3,
$$

where the symbols $L_{a b}^{c}$ are defined by

$$
\nabla_{E_{a}} E_{b}=\sum_{c=1}^{3} \frac{L_{a b}^{c}}{2} E_{c}, \quad a, b=1,2,3 .
$$

Let $C_{a b}^{c}$ be the structure constants of the Lie algebra of $M$, i.e., $\left[E_{a}, E_{b}\right]=\sum_{c=1}^{3} C_{a b}^{c} E_{c}$. Therefore, by the Levi-Civita Theorem, we have

$$
L_{a b}^{c}=\left(C_{a b}^{c}-C_{b c}^{a} \varepsilon_{a} \varepsilon_{c}-C_{a c}^{b} \varepsilon_{b} \varepsilon_{c}\right),
$$

where $\varepsilon_{a}=\left\langle E_{a}, E_{a}\right\rangle$, with $a=1,2,3$.

Consequently, in the case of three-dimensional Lie groups, the Theorem 1 may be written as follows.

Theorem 2 ([17]) Let $M$ be a three-dimensional Lie group endowed with a left-invariant Lorentzian metric and let $\left\{E_{1}, E_{2}, E_{3}\right\}$ be a left-invariant orthonormal frame field. Let $f$ : $\Omega \rightarrow M$ be a conformal minimal immersion, where $\Omega \subset \mathbb{K}$ is an open set. We denote by $\phi \in \Gamma\left(f^{*} T M \otimes \mathbb{K}\right)$ the (para)complex tangent vector

$$
\phi(z)=\frac{\partial f}{\partial z} .
$$

Then, the components $\psi_{a}, a=1,2,3$, of $\phi$ defined by

$$
\phi(z)=\left.\sum_{a=1}^{3} \psi_{a} E_{a}\right|_{f(z)},
$$

satisfy the followings conditions:

(i) $\left|\psi_{1}\right|^{2}+\left|\psi_{2}\right|^{2}-\left|\psi_{3}\right|^{2} \neq 0$,

(ii) $\psi_{1}^{2}+\psi_{2}^{2}-\psi_{3}^{2}=0$,

(iii) $\frac{\partial \psi_{c}}{\partial \bar{z}}+\sum_{a, b=1}^{3} \frac{L_{a b}^{c}}{2} \bar{\psi}_{a} \psi_{b}=0$,

where the derivative with respect to $\bar{z}$ is in the classical sense in the spacelike case and in the Lorentz sense in the timelike case.

Conversely, if $\Omega \subset \mathbb{K}$ is a simply connected domain and $\psi_{a}: \Omega \rightarrow \mathbb{K}, a=1,2,3$, are (para)complex functions satisfying the conditions above, then the map $f: \Omega \rightarrow M$ which coordinate functions are given by:

$$
f_{a}=2 \mathcal{R} e \int \sum_{b=1}^{3} A_{a b} \psi_{b} d z, \quad a=1,2,3,
$$

is a well-defined conformal minimal immersion. 
Remark 3 We observe explicitly that the equations above do not give the coordinate functions of the immersion $f$ just by a direct integration since the functions $A_{a b}$ must be computed along the solutions $f_{a}$. The formula (8) is in fact an integral equation. However, as we will see, for special ambient manifolds this problem can be avoided by ad hoc arguments.

\section{The Björling problem for three-dimensional Lie groups}

We denote by $M$ a three-dimensional Lie group endowed with a left-invariant Lorentzian metric $g$. Let $\beta: I \rightarrow M$ be an analytic curve in $M$ and $V: I \rightarrow T M$ a unitary analytic spacelike (respectively, timelike) vector field along $\beta$, such that $g(\dot{\beta}, V) \equiv 0$. The Björling problem can be formulated as follows:

Determine a timelike (respectively, spacelike) minimal surface

$$
f: I \times(-\epsilon, \epsilon)=\Omega \subseteq \mathbb{K} \rightarrow M
$$

such that

i. $f(u, 0)=\beta(u)$,

ii. $N(u, 0)=V(u)$,

for all $u \in I$, where $N: \Omega \rightarrow T M$ is the Gauss map of the surface.

Before showing that the above problem has a unique solution, we prove the following:

Lemma 1 Let $\psi_{i}: \Omega \subseteq \mathbb{K} \rightarrow \mathbb{K}, i=1,2$, be two differentiable functions and $\psi_{3}^{2}=$ $\psi_{1}^{2}+\psi_{2}^{2}$. We suppose that $\psi_{i}, i=1,2$, satisfy the two first equations of the third item in Theorem 2. Then, $\psi_{3}$ satisfies the third equation.

Proof Differentiating $\psi_{3}^{2}=\psi_{1}^{2}+\psi_{2}^{2}$ with respect to $\bar{z}$ and using that $\psi_{i}, i=1,2$, satisfy the two first equations of item (iii) in Theorem 2, we have that

$$
\psi_{3} \frac{\partial \psi_{3}}{\partial \bar{z}}=\psi_{1} \frac{\partial \psi_{1}}{\partial \bar{z}}+\psi_{2} \frac{\partial \psi_{2}}{\partial \bar{z}}=-\frac{1}{2} \sum_{j, k=1}^{3}\left[L_{j k}^{1} \psi_{1}+L_{j k}^{2} \psi_{2}\right] \bar{\psi}_{j} \psi_{k} .
$$

Therefore, to prove the lemma, it suffices to show that

$$
\sum_{j, k=1}^{3}\left[L_{j k}^{1} \psi_{1}+L_{j k}^{2} \psi_{2}-L_{j k}^{3} \psi_{3}\right] \bar{\psi}_{j} \psi_{k}=0
$$

We may write the above sum as follows:

$$
\begin{aligned}
& \sum_{i=1}^{3}\left\{L_{i 1}^{1} \psi_{1}^{2}+L_{i 2}^{2} \psi_{2}^{2}-L_{i 3}^{3} \psi_{3}^{2}+\left(L_{i 1}^{2}+L_{i 2}^{1}\right) \psi_{1} \psi_{2}\right. \\
& \left.\quad+\left(L_{i 3}^{1}-L_{i 1}^{3}\right) \psi_{1} \psi_{3}+\left(L_{i 3}^{2}-L_{i 2}^{3}\right) \psi_{3} \psi_{2}\right\} \bar{\psi}_{i} .
\end{aligned}
$$

Now, using (7), we have that

$$
L_{i k}^{k}=L_{i 1}^{2}+L_{i 2}^{1}=L_{i 3}^{1}-L_{i 1}^{3}=L_{i 3}^{2}-L_{i 2}^{3}=0, \quad i=1,2,3 .
$$

Then,

$$
\frac{\partial \psi_{3}}{\partial \bar{z}}+\frac{1}{2} \sum_{j, k=1}^{3} L_{j k}^{3} \bar{\psi}_{j} \psi_{k}=0
$$


4.1 The Björling problem for timelike surfaces

We observe that if $f$ is a timelike conformal minimal immersion we have that $\psi_{i}=\frac{\partial f_{i}}{\partial z}$, $i=1,2,3$, satisfy the condition ( $i$ ii $)$ in Theorem 2 , which is equivalent to the hyperbolic system of partial differential equations (see [10]):

$$
\frac{\partial^{2} f_{i}}{\partial u^{2}}-\frac{\partial^{2} f_{i}}{\partial v^{2}}+B_{i}\left(\frac{\partial f_{i}}{\partial u}, \frac{\partial f_{i}}{\partial v}\right)=0
$$

where $B_{i}, i=1,2,3$, contain at most first-order derivatives of the functions $f_{i}$. So the Björling problem may be interpreted as a Cauchy problem involving quasilinear partial differentiable equations (9).

Remark 4 Let $\gamma(s)=(u(s), v(s))$ be a characteristic curve in $\Omega$ (see [10]), then

$$
u^{\prime}(s)^{2}-v^{\prime}(s)^{2}=0
$$

that is, $\gamma$ is a straight line parallel to $u= \pm v$ in $\Omega$.

It is known that the Cauchy problem may not have a unique solution, or it does not have solutions at all if the initial data is along characteristic curves. Moreover, these lines correspond to the lightlike curves of the Björling problem. Consequently, we consider two cases: When $\beta$ is a timelike curve, we will call the corresponding problem the timelike Björling problem, and when $\beta$ is a spacelike curve, we will have the spacelike Björling problem.

Therefore, the Björling problem is a Cauchy problem with initial data:

$$
f_{i}(u, 0)=\beta_{i}(u), \quad(V(u) \times \dot{\beta}(u))_{i}= \pm\left(\frac{\partial f}{\partial v}(u, 0)\right)_{i}, \quad i=1,2,3,
$$

where in the above equation we have the sign "+" (respectively, "-") if $\beta$ is a timelike (respectively, spacelike) curve.

Theorem 3 (Timelike Björling problem) Let $\beta: I \rightarrow M$ be an analytic timelike curve in $M$ and $V: I \rightarrow T M$ a unitary analytic spacelike vector field along $\beta$, such that $g(\dot{\beta}, V) \equiv 0$. Then, there exists an analytic conformal timelike minimal surface

$$
f: I \times(-\epsilon, \epsilon)=\Omega \subseteq \mathbb{L} \rightarrow M
$$

such that

i. $f(u, 0)=\beta(u)$,

ii. $N(u, 0)=V(u)$,

for all $u \in I$, where $N: \Omega \rightarrow T M$ is the Gauss map of the surface. Moreover two such surfaces coincide along the intersection of their domains.

Proof Consider the system

$$
\left\{\begin{array}{l}
\frac{\partial \psi_{1}}{\partial \bar{z}}+\frac{1}{2} \sum_{j, k=1}^{3} L_{j k}^{1} \bar{\psi}_{j} \psi_{k}=0 \\
\frac{\partial \psi_{2}}{\partial \bar{z}}+\frac{1}{2} \sum_{j, k=1}^{3} L_{j k}^{2} \bar{\psi}_{j} \psi_{k}=0
\end{array}\right.
$$


where $\psi_{i}: \Omega \rightarrow \mathbb{L}$ and $\psi_{3}^{2}=\psi_{1}^{2}+\psi_{2}^{2}$.

As $\beta$ is a timelike curve, then it is not a characteristic curve and, so, this system is of Cauchy-Kovalevskaya type ([21]). Therefore, fixing the initial data, it admits a unique analytic solution (locally). Hence, we must find the initial data so that the minimal surface has the required properties. We observe that, if $f$ is a solution of the Björling problem, we have that

$$
\frac{\partial f}{\partial u}(u, 0)=\dot{\beta}(u) \text { and } \frac{\partial f}{\partial v}(u, 0)=V(u) \times \dot{\beta}(u) .
$$

Then,

$$
\phi(u, 0)=\frac{1}{2}\left(\frac{\partial f}{\partial u}+\tau \frac{\partial f}{\partial v}\right)(u, 0)=\frac{1}{2}(\dot{\beta}(u)+\tau V(u) \times \dot{\beta}(u)) .
$$

So the initial condition for the system is given by

$$
\psi(u, 0)=A^{-1}(\beta(u)) \phi(u, 0),
$$

where $A$ is given in (6).

We note that Lemma 1 implies that the functions $\psi_{i}$ satisfy the equations (ii) and (iii) of Theorem 2. Furthermore, from (12), it follows that

$$
\langle\langle\phi(u, 0), \phi(u, 0)\rangle\rangle=\frac{1}{4}[g(\dot{\beta}, \dot{\beta})-g(V \times \dot{\beta}, V \times \dot{\beta})]<0,
$$

because, from (5), it results that $g(V \times \dot{\beta}, V \times \dot{\beta})=-g(\dot{\beta}, \dot{\beta})$ and $\beta$ is a timelike curve. Shrinking $\Omega$ if necessary, we can assume that

$$
\langle\langle\phi(u, v), \phi(u, v)\rangle\rangle<0, \quad(u, v) \in \Omega .
$$

As $A$ is the Jacobian matrix of a left-invariant translation in $M$, it results that

$$
\left|\psi_{1}(u, v)\right|^{2}+\left|\psi_{2}(u, v)\right|^{2}-\left|\psi_{3}(u, v)\right|^{2}=\langle\langle\phi(u, v), \phi(u, v)\rangle\rangle<0, \quad(u, v) \in \Omega .
$$

Therefore, the functions $\psi_{i}, i=1,2,3$ satisfy the conditions of Theorem 2. So, from the Cauchy-Kovalevskaya Theorem, there exists a unique analytic conformal timelike minimal immersion, which is a local solution of the timelike Björling problem. Observe that the initial condition forces the choice of one of the determinations of $\psi_{3}^{2}=\psi_{1}^{2}+\psi_{2}^{2}$.

Up to now we have proved the existence and uniqueness of a local solution. We may consider that $I$ is compact. Using compactness of $I$ and local uniqueness, in the case of $\beta(I)$ is contained in a coordinate neighborhood, for $\epsilon>0$ sufficiently small, we can prove (immediately) existence and uniqueness of the solution. In the general case, as $I$ is compact, we can cover it with a finite number of inverse images, via $\beta$, of coordinate neighborhoods, and using (again) the local uniqueness of the problem, we obtain the global solution.

We can prove that the spacelike Björling problem has a unique analytic solution analogously to the timelike case. In this case, the initial data are

$$
\psi(u, 0)=A^{-1}(\beta(u)) \phi(u, 0),
$$

where

$$
\phi(u, 0)=\frac{1}{2}(\dot{\beta}(u)-\tau V(u) \times \dot{\beta}(u)) .
$$

Theorem 4 (Spacelike Björling problem) Let $\beta: I \rightarrow M$ be an analytic spacelike curve in $M$ and $V: I \rightarrow T M$ a unitary analytic spacelike vector field along $\beta$, such that $g(\dot{\beta}, V) \equiv 0$. Then, there exists an analytic conformal timelike minimal surface

$$
f: I \times(-\epsilon, \epsilon)=\Omega \subseteq \mathbb{L} \rightarrow M
$$


such that:

i. $f(u, 0)=\beta(u)$,

ii. $N(u, 0)=V(u)$,

for all $u \in I$, where $N: \Omega \rightarrow T M$ is the Gauss map of the surface. Moreover, two such surfaces coincide along the intersection of their domains.

\subsection{The Björling problem for spacelike surfaces}

The Björling problem for spacelike surfaces has a unique solution, and the proof is analogous to the case of the timelike surfaces. In this case, the system of condition (iii) in Theorem 2 is equivalent to the elliptic system of partial differential equations (see [10]):

$$
\frac{\partial^{2} f_{i}}{\partial u^{2}}+\frac{\partial^{2} f_{i}}{\partial v^{2}}+B_{i}\left(\frac{\partial f_{i}}{\partial u}, \frac{\partial f_{i}}{\partial v}\right)=0
$$

where $B_{i}, i=1,2,3$ contain at most first-order derivatives of the functions $f_{i}$.

So the Björling problem may be interpreted as a Cauchy problem involving quasilinear partial differentiable equations (13) with initial data:

$$
f_{i}(u, 0)=\beta_{i}(u), \quad(V(u) \times \dot{\beta}(u))_{i}=-\left(\frac{\partial f}{\partial v}(u, 0)\right)_{i}, \quad i=1,2,3 .
$$

Therefore, we can use again the Cauchy-Kovalevskaya Theorem (see [10]) to show that the problem has a unique analytic solution with the initial data given by

$$
\psi(u, 0)=A^{-1}(\beta(u)) \phi(u, 0),
$$

where

$$
\phi(u, 0)=\frac{1}{2}(\dot{\beta}(u)+i V(u) \times \dot{\beta}(u)) .
$$

Theorem 5 (Björling problem) Let $\beta: I \rightarrow M$ be an analytic spacelike curve in $M$ and $V: I \rightarrow T M$ a unitary analytic timelike vector field along $\beta$, such that $g(\dot{\beta}, V) \equiv 0$. Then, there exists an analytic conformal spacelike minimal surface

$$
f: I \times(-\epsilon, \epsilon)=\Omega \subseteq \mathbb{C} \rightarrow M
$$

such that:

i. $f(u, 0)=\beta(u)$,

ii. $N(u, 0)=V(u)$,

for all $u \in I$, where $N: \Omega \rightarrow T M$ is the Gauss map of the surface. Moreover, two such surfaces coincide along the intersection of their domains.

Now we will construct some examples of minimal surfaces in the Heisenberg group $\mathbb{H}_{3}$, in the de Sitter space $\mathbb{S}_{1}^{3}$ and in the space $\mathbb{H}^{2} \times \mathbb{R}$, equipped with left-invariant Lorentzian metrics.

\section{The Lorentzian Heisenberg group $\mathbb{H}_{3}$}

We consider the Heisenberg group

$$
\mathbb{H}_{3}=\left\{\left[\begin{array}{ccc}
1 & x & z+\frac{1}{2} x y \\
0 & 1 & y \\
0 & 0 & 1
\end{array}\right]: x, y, z \in \mathbb{R}\right\},
$$


equipped with the left-invariant Lorentzian metric given by

$$
g=d x^{2}+d y^{2}-\left(\frac{1}{2} y d x-\frac{1}{2} x d y+d z\right)^{2} .
$$

With respect to $g$, the left-invariant frame field given by

$$
E_{1}=\frac{\partial}{\partial x}-\frac{y}{2} \frac{\partial}{\partial z}, \quad E_{2}=\frac{\partial}{\partial y}+\frac{x}{2} \frac{\partial}{\partial z}, \quad E_{3}=\frac{\partial}{\partial z},
$$

is orthonormal, $\left\{E_{1}, E_{2}\right\}$ are spacelike and $E_{3}$ is timelike. Also, the matrix $A$ is

$$
A=\left[\begin{array}{ccc}
1 & 0 & 0 \\
0 & 1 & 0 \\
-\frac{y}{2} & \frac{x}{2} & 1
\end{array}\right]
$$

and the nonzero $L_{i j}^{k}$ are $L_{12}^{3}=L_{13}^{2}=L_{31}^{2}=\frac{1}{2}$ and $L_{21}^{3}=L_{32}^{1}=L_{23}^{1}=-\frac{1}{2}$. Consequently, the system (10) becomes

$$
\left\{\begin{array}{l}
\frac{\partial \psi_{1}}{\partial \bar{z}}-\mathcal{R} e\left(\bar{\psi}_{3} \psi_{2}\right)=0, \\
\frac{\partial \psi_{2}}{\partial \bar{z}}+\mathcal{R} e\left(\bar{\psi}_{3} \psi_{1}\right)=0 .
\end{array}\right.
$$

Then, the coordinate functions of the minimal immersion $f$ are given by

$$
\left\{\begin{array}{l}
f_{1}=2 \mathcal{R} e \int \psi_{1} d z \\
f_{2}=2 \mathcal{R} e \int \psi_{2} d z \\
f_{3}=2 \mathcal{R} e \int\left(\frac{f_{1}}{2} \psi_{2}-\frac{f_{2}}{2} \psi_{1}+\psi_{3}\right) d z .
\end{array}\right.
$$

So, knowing the $\psi_{i}, i=1,2,3$, that are solutions of a constant coefficients PDE, we can compute $f_{1}, f_{2}$ by integration and, substituting in the third equation of (15), we can compute $f_{3}$ by direct integration (see Remark 3 ).

Example 1 ( The timelike vertical plane $y=c$, timelike case) First of all, we consider the curve

$$
\beta(u)=\left(\cosh u, c,-\frac{c}{2} \cosh u+\sinh u\right), \quad u \in \mathbb{R}, \quad c \in \mathbb{R},
$$

and the unit vector field $V(u)=E_{2}(\beta(u))$. Since

$$
\dot{\beta}(u)=\sinh u E_{1}+\cosh u E_{3},
$$

then $g(\dot{\beta}, \dot{\beta})=-1$. Moreover, $g(V, V)=1$ and $g(\dot{\beta}, V)=0$. Thus, we have a timelike Björling problem. As

$$
E_{2} \times E_{1}=E_{3} \text { and } E_{2} \times E_{3}=E_{1},
$$

we have that $V(u) \times \dot{\beta}(u)=\cosh u E_{1}+\sinh u E_{3}$. So

$$
\phi(u, 0)=\frac{1}{2}\left[(\sinh u+\tau \cosh u) E_{1}+(\cosh u+\tau \sinh u) E_{3}\right] .
$$

Consequently,

$$
\psi(u, 0)=A^{-1}(\beta(u)) \phi(u, 0)=\frac{1}{2}(\sinh u+\tau \cosh u, 0, \cosh u+\tau \sinh u) .
$$


Therefore, the solution of system (14), which satisfies the initial condition (16), is

$$
\left\{\begin{array}{l}
\psi_{1}(u, v)=\frac{e^{v}}{2}(\sinh u+\tau \cosh u), \\
\psi_{2}(u, v)=0 \\
\psi_{3}(u, v)=\frac{e^{v}}{2}(\cosh u+\tau \sinh u) .
\end{array}\right.
$$

Furthermore, (17) satisfy the conditions of Theorem 3. Then, we obtain the conformal timelike minimal immersion

$$
f(u, v)=\left(e^{v} \cosh u, c, e^{v}\left(-\frac{c}{2} \cosh u+\sinh u\right)\right)
$$

that is a timelike vertical plane $y=c$, and it represents the solution of the Björling problem for the given pair $(\beta, V)$.

Example 2 [Helicoids] Consider $\beta(u)=(\rho(u), 0, b), b \in \mathbb{R}$ and

$$
V(u)=\frac{\rho^{2}(u)-2 c}{2 \rho^{\prime}(u)} E_{2}-\frac{\rho(u)}{\rho^{\prime}(u)} E_{3}, \quad c \in \mathbb{R},
$$

where $\rho(u), u \in(a, d) \subset \mathbb{R}$, is a real function satisfying

$$
\sqrt{\left(\rho^{\prime}\right)^{2}+\rho^{2}}=\frac{\rho^{2}}{2}-c .
$$

As $\dot{\beta}(u)=\rho^{\prime}(u) E_{1}$, then $g(\dot{\beta}, \dot{\beta})=\rho^{\prime 2}$. Moreover,

$$
g(V, V)=1, \quad g(\dot{\beta}, V)=0 .
$$

Thus, we have a spacelike Björling problem. Since $E_{2} \times E_{1}=E_{3}$ and $E_{3} \times E_{1}=E_{2}$, we obtain

$$
V(u) \times \dot{\beta}(u)=\frac{\rho^{2}(u)-2 c}{2} E_{3}-\rho(u) E_{2} .
$$

Then,

$$
\phi(u, 0)=\left(\frac{\rho^{\prime}(u)}{2}, \frac{\tau \rho(u)}{2}, \frac{c \tau}{2}\right)
$$

and, so, it follows that

$$
\psi(u, 0)=\left(\frac{\rho^{\prime}(u)}{2}, \frac{\tau \rho(u)}{2},-\frac{\tau\left(\rho^{2}(u)-2 c\right)}{4}\right) .
$$

Therefore, the solution of (14), which satisfies the initial condition (19), is

$$
\left\{\begin{array}{l}
\psi_{1}(u, v)=\frac{1}{2}\left(\rho^{\prime}(u) \cos v-\tau \rho(u) \sin v\right), \\
\psi_{2}(u, v)=\frac{1}{2}\left(\rho^{\prime}(u) \sin v+\tau \rho(u) \cos v\right), \\
\psi_{3}(u, v)=-\frac{\tau}{4}\left(\rho^{2}(u)-2 c\right) .
\end{array}\right.
$$

Furthermore, (20) satisfy the conditions of Theorem 4. Then, integrating we obtain the following solution of the Björling problem:

$$
f(u, v)=(\rho(u) \cos v, \rho(u) \sin v, c v+b),
$$

which represents a timelike helicoid if $c \neq 0$, and the horizontal plane $z=b$, if $c=0$. 
Example 3 [The saddle-type surface] Consider $\beta(u)=(4 c u,-4 Q(0),-8 c u Q(0))$ and

$$
V(u)=-\frac{4 c Q(0)}{Q^{\prime}(0)} E_{1}+\frac{c}{Q^{\prime}(0)} E_{3}, \quad u \in(a, b) \subset \mathbb{R}, \quad c \in \mathbb{R},
$$

where $Q(v)$ is a real differentiable function with $Q^{\prime}(v) \neq 0$, for all $v \in \mathbb{R}$, which satisfies

$$
4 c Q(v)=\sqrt{Q^{\prime}(v)^{2}+c^{2}} \text {. }
$$

As

$$
\dot{\beta}(u)=4 c E_{1}-16 c Q(0) E_{3},
$$

then $g(\dot{\beta}(u), \dot{\beta}(u))=-16 Q^{\prime}(0)^{2}$. Moreover, $g(V(u), V(u))=1$ and $g(\dot{\beta}(u), V(u))=0$. Thus, we have a timelike Björling problem. Since

$$
V(u) \times \dot{\beta}(u)=-4 Q^{\prime}(0) E_{2},
$$

then

$$
\phi(u, 0)=\left(2 c,-2 Q^{\prime}(0) \tau,-4 c Q(0)-4 a u Q^{\prime}(0) \tau\right)
$$

and, so, it follows that

$$
\psi(u, 0)=\left(2 c,-2 Q^{\prime}(0) \tau,-8 c Q(0)\right) .
$$

Therefore, the solution of (14), which satisfies the initial condition (21), is

$$
\left\{\begin{array}{l}
\psi_{1}(u, v)=2 c, \\
\psi_{2}(u, v)=-2 \tau Q^{\prime}(v), \\
\psi_{3}(u, v)=-8 c Q(v) .
\end{array}\right.
$$

Furthermore, (22) satisfy the conditions of the Theorem 3. Then, integrating we obtain

$$
f(u, v)=(4 c u,-4 Q(v),-8 c u Q(v)),
$$

whose image lies on the graph of the function $z=\frac{1}{2} x y$.

\section{The de Sitter space $\mathbb{S}_{1}^{3}$}

The de Sitter space $\mathbb{S}_{1}^{3}$ might be modeled as the halfspace

$$
\mathbb{R}_{+}^{3}=\left\{\left(x_{1}, x_{2}, x_{3}\right) \in \mathbb{R}^{3}: x_{3}>0\right\}
$$

endowed with the left-invariant Lorentzian metric given by

$$
g=\frac{1}{x_{3}^{2}}\left(d x_{1}^{2}+d x_{2}^{2}-d x_{3}^{2}\right) .
$$

An orthonormal basis of left-invariant vector fields is given by

$$
E_{1}=x_{3} \frac{\partial}{\partial x_{1}}, \quad E_{2}=x_{3} \frac{\partial}{\partial x_{2}}, \quad E_{3}=x_{3} \frac{\partial}{\partial x_{3}},
$$

where $\left\{E_{1}, E_{2}\right\}$ are spacelike and $E_{3}$ timelike. Then,

$$
A=\left[\begin{array}{ccc}
x_{3} & 0 & 0 \\
0 & x_{3} & 0 \\
0 & 0 & x_{3}
\end{array}\right]
$$


The only $L_{i j}^{k}$ nonzero are $L_{13}^{1}=L_{23}^{2}=L_{11}^{3}=L_{22}^{3}=-1$. So (10) becomes

$$
\left\{\begin{array}{l}
\frac{\partial \psi_{1}}{\partial \bar{z}}-\bar{\psi}_{1} \psi_{3}=0 \\
\frac{\partial \psi_{2}}{\partial \bar{z}}-\bar{\psi}_{2} \psi_{3}=0
\end{array}\right.
$$

Therefore, the conformal minimal immersion $f$ is given by

$$
\left\{\begin{array}{l}
f_{1}=2 \mathcal{R} e \int f_{3} \psi_{1} d z, \\
f_{2}=2 \mathcal{R} e \int f_{3} \psi_{2} d z, \\
f_{3}=\exp \left(2 \mathcal{R} e \int \psi_{3} d z\right) .
\end{array}\right.
$$

Again, knowing the $\psi_{i}, i=1,2,3$, we can compute $f_{3}$ by direct integration and, substituting in the first two equations, we can also compute $f_{1}, f_{2}$ by direct integration (see, again, Remark 3).

Example 4 (Timelike vertical plane $y=c$, the spacelike case) Consider

$$
\beta(u)=(\sinh u, c, \cosh u), \quad u \in \mathbb{R}
$$

and $V(u)=E_{2}$. We have that

$$
\dot{\beta}(u)=E_{1}+\frac{\sinh u}{\cosh u} E_{3}, \quad g(\dot{\beta}(u), \dot{\beta}(u))=\frac{1}{\cosh ^{2}(u)}>0 .
$$

Furthermore, $g(V, V)=1$ and $g(\dot{\beta}, V)=0$. Thus, the pair $(\beta, V)$ produces a spacelike Björling problem. Since

$$
V(u) \times \dot{\beta}(u)=E_{3}+\frac{\sinh u}{\cosh u} E_{1}=(\sinh u, 0, \cosh u),
$$

we obtain that

$$
\phi(u, 0)=\frac{1}{2}(\dot{\beta}(u)-\tau V(u) \times \dot{\beta}(u))=\frac{1}{2}(\cosh u-\tau \sinh u, 0, \sinh u-\tau \cosh u) .
$$

Therefore,

$$
\psi(u, 0)=A^{-1}(\beta(u)) \phi(u, 0)=\frac{1}{2}\left(\frac{\cosh u-\tau \sinh u}{\cosh u}, 0, \frac{\sinh u-\tau \cosh u}{\cosh u}\right) .
$$

As $\psi(u, 0)$ is a solution of (23), the uniqueness implies that $\psi(u, v)=\psi(u, 0)$. So the conformal timelike minimal immersion is given by

$$
f(u, v)=\left(e^{-v} \sinh u, c, e^{-v} \cosh u\right) .
$$

Example 5 Consider

$$
\beta(u)=\left(\frac{1}{\sqrt{2}} \sinh u, \frac{1}{\sqrt{2}} \sinh u, \cosh u\right), \quad V(u)=-\frac{E_{1}}{\sqrt{2}}+\frac{E_{2}}{\sqrt{2}} .
$$

As

$$
\dot{\beta}(u)=\frac{E_{1}}{\sqrt{2}}+\frac{E_{2}}{\sqrt{2}}+\frac{\sinh u}{\cosh u} E_{3}
$$


and

$$
g(\dot{\beta}(u), \dot{\beta}(u))=\frac{1}{\cosh ^{2}(u)},
$$

then, we have a spacelike Björling problem. Also,

$$
g(V(u), V(u))=1, \quad g(\dot{\beta}(u), V(u))=0
$$

and

$$
V(u) \times \dot{\beta}(u)=\left(\frac{1}{\sqrt{2}} \sinh u, \frac{1}{\sqrt{2}} \sinh u, \cosh u\right) .
$$

Therefore,

$$
\phi(u, 0)=\frac{1}{2}\left(\frac{1}{\sqrt{2}}(\cosh u-\tau \sinh u), \frac{1}{\sqrt{2}}(\cosh u-\tau \sinh u), \sinh u-\tau \cosh u\right)
$$

and

$\psi(u, 0)=A^{-1}(\beta(u)) \phi(u, 0)=\frac{1}{2}\left(\frac{\cosh u-\tau \sinh u}{\sqrt{2} \cosh u}, \frac{\cosh u-\tau \sinh u}{\sqrt{2} \cosh u}, \frac{\sinh u-\tau \cosh u}{\cosh u}\right)$.

Since $\psi(u, 0)$ is solution of (23), the uniqueness implies that $\psi(u, v)=\psi(u, 0)$. Integrating (24), it results that

$$
f(u, v)=e^{-v}\left(\frac{\sinh u}{\sqrt{2}}, \frac{\sinh u}{\sqrt{2}}, \cosh u\right) .
$$

\section{The space $\mathbb{H}^{2} \times \mathbb{R}$}

We consider the hyperbolic space $\mathbb{H}^{2}$ modeled as the halfspace

$$
\mathbb{R}_{+}^{2}=\left\{\left(x_{1}, x_{2}\right) \in \mathbb{R}^{2}: x_{2}>0\right\}
$$

and endowed with the left-invariant metric given by

$$
g_{\mathbb{H}}=\frac{1}{x_{2}^{2}}\left(d x_{1}^{2}+d x_{2}^{2}\right) .
$$

The space $\mathbb{H}^{2} \times \mathbb{R}$ is a Lie group with the product structure and the product metric

$$
g=g_{\mathbb{H}}-d x_{3}^{2}
$$

is a left-invariant Lorentzian metric. An orthonormal basis of left-invariant vector fields is given by

$$
E_{1}=x_{2} \frac{\partial}{\partial x_{1}}, \quad E_{2}=x_{2} \frac{\partial}{\partial x_{2}}, \quad E_{3}=\frac{\partial}{\partial x_{3}}
$$

where $\left\{E_{1}, E_{2}\right\}$ are spacelike and $E_{3}$ timelike. Then,

$$
A=\left[\begin{array}{ccc}
x_{2} & 0 & 0 \\
0 & x_{2} & 0 \\
0 & 0 & 1
\end{array}\right]
$$


The only $L_{i j}^{k}$ nonzero are $L_{12}^{1}=-2$ and $L_{11}^{2}=2$. So (10) becomes

$$
\left\{\begin{array}{l}
\frac{\partial \psi_{1}}{\partial \bar{z}}-\psi_{1} \bar{\psi}_{2}=0 \\
\frac{\partial \psi_{2}}{\partial \bar{z}}+\bar{\psi}_{1} \psi_{1}=0
\end{array}\right.
$$

Therefore, the conformal minimal immersion $f$ is given by

$$
\left\{\begin{array}{l}
f_{1}(u, v)=2 \mathcal{R} e \int f_{2} \psi_{1} d z \\
f_{2}(u, v)=\exp \left(2 \mathcal{R} e \int \psi_{2} d z\right) \\
f_{3}(u, v)=2 \mathcal{R} e \int \psi_{3} d z
\end{array}\right.
$$

Again, knowing the $\psi_{i}, i=1,2,3$, we can compute $f_{2}$ and $f_{3}$ by direct integration and, substituting in the first equation, we can also compute $f_{1}$ by direct integration (see, again, Remark 3)

We observe that if $\psi_{2}$ is a $\mathbb{L}$-differentiable (or holomorphic) function, then from (25) it follows that $\psi_{1} \bar{\psi}_{1}=0$ and $\psi_{1} \frac{\partial \psi_{1}}{\partial \bar{z}}=0$. For example, if $\psi_{1}=0$, we get the planes $x_{1}=$ cte.

Example 6 (Spacelike horizontal plane $z=c$ ) Consider

$$
\beta(u)=(\cos u, \sin u, c), \quad u \in(0, \pi)
$$

and $V(u)=E_{3}$. We have that

$$
\dot{\beta}(u)=-E_{1}+\frac{\cos u}{\sin u} E_{2}, \quad g(\dot{\beta}(u), \dot{\beta}(u))=\frac{1}{\sin ^{2}(u)}>0 .
$$

Furthermore, $g(V, V)=-1$ and $g(\dot{\beta}, V)=0$. Thus, the pair $(\beta, V)$ produces a Björling problem for spacelike surfaces. As

$$
V(u) \times \dot{\beta}(u)=-E_{2}-\frac{\cos u}{\sin u} E_{1}=(-\cos u,-\sin u, 0),
$$

we obtain that

$$
\phi(u, 0)=\frac{1}{2}(\dot{\beta}(u)+i V(u) \times \dot{\beta}(u))=\frac{1}{2}(-\sin u-i \cos u, \cos u-i \sin u, 0) .
$$

Therefore,

$$
\psi(u, 0)=A^{-1}(\beta(u)) \phi(u, 0)=\frac{1}{2}\left(-\frac{\sin u+i \cos u}{\sin u}, \frac{\cos u-i \sin u}{\sin u}, 0\right) .
$$

Since $\psi(u, 0)$ is a solution of $(25)$, the uniqueness implies that $\psi(u, v)=\psi(u, 0)$. Consequently, we have the conformal spacelike minimal immersion given by

$$
f(u, v)=\left(e^{v} \cos u, e^{v} \sin u, c\right) .
$$

Acknowledgments The authors would like to thank the referee for his suggestions. 


\section{References}

1. Aledo, J., Chaves, R.M.B., Gálvez, J.A.: The Cauchy problem for improper affine spheres and the Hessian one equation. Trans. Am. Math. Soc. 359, 4183-4208 (2007)

2. Aledo, J., Gálvez, J.A., Mira, P.: A D’Alembert formula for flat surfaces in the 3-sphere. J. Geom. Anal. 19, 211-232 (2009)

3. Alías, L.J., Chaves, R.M.B., Mira, P.: Björling problem for maximal surfaces in Lorentz-Minkowski space. Math. Proc. Camb. Phil. Soc. 134(2), 289-316 (2003)

4. Asperti, A., Vilhena, J.M.: Björling problem for spacelike, zero mean curvature surfaces in $\mathbb{L}^{4}$. J. Geom. Phys. 56(2), 196-213 (2006)

5. Björling, E.G.: In integrazionem aequationis derivatarum partialum superfici cujus inpuncto uniquoque principales ambos radii curvedinis aequales sunt sngoque contrario. Arch. Math. Phys. 4(1), 290-315 (1844)

6. Brander, D., Dorfmeister, J.F.: The Björling problem for non-minimal constant mean curvature surfaces. Comm. Anal. Geom. 18, 171-194 (2010)

7. Brander, D., Svensson, M.: The geometric Cauchy problem for surfaces with Lorentzian harmonic Gauss maps. J. Differ. Geom. 93, 37-66 (2013)

8. Chaves, R.M.B., Dussan, M.P., Magid, M.: Björling problem for timelike surfaces in the LorentzMinkowski space. J. Math. Anal. Appl. 337, 481-494 (2011)

9. Dussan, M.P., Magid, M.: The Björling problem for timelike surfaces in $\mathbb{R}_{2}^{4}$. J. Geom. Phys. 73, 187-199 (2013)

10. Evans, L.C.: Partial Differential Equations. AMS, Providence (1998)

11. Gálvez, J.A., Mira, P.: Dense solutions to the Cauchy problem for minimal surfaces. Bull. Braz. Math. Soc. (N.S.) 35, 387-394 (2004)

12. Gálvez, J.A., Mira, P.: Embedded isolated singularities of flat surfaces in hyperbolic 3-space. Calc. Var. Partial Differ. Equ. 24, 239-260 (2005)

13. Gálvez, J.A., Mira, P.: The Cauchy problem for the Liouville equation and Bryant surfaces. Adv. Math. 195, 456-490 (2005)

14. Kobayashi, O.: Maximal surfaces in 3-dimensional Lorentz space $\mathbb{L}^{3}$. Tokyo J. Math. 6, 297-309 (1983)

15. Kokubu, M.: Weierstrass representation for minimal surfaces in hyperbolic space. Tôhoku Math. J. 49, 367-377 (1997)

16. Konderak, J.: A Weierstrass representation theorem for Lorentz surfaces. Complex Var. Theory Appl. 50(5), 319-332 (2005)

17. Lira, J.H., Melo, M., Mercuri, F.: A Weierstrass representation for minimal surfaces in 3-dimensional manifolds. Results. Math. 60, 311-323 (2011)

18. Mercuri, F., Montaldo, S., Piu, P.: Weierstrass representation formula of minimal surfaces in $\mathbb{H}_{3}$ and $\mathbb{H}^{2} \times \mathbb{R}$. Acta Math. Sinica 22, 1603-1612 (2006)

19. Mercuri, F., Irene, I., Onnis, : On the Björling problem in a 3-dimensional Lie group. Illinois J. Math. 53(2), 431-440 (2009)

20. Mira, P.: Complete minimal Möbius strips in $\mathbb{R}^{n}$ and the Björling problem. J. Geom. Phys. 56, 1506-1515 (2006)

21. Petrovsky, I.G.: Lectures on Partial Differential Equations. Interscience Publishers, New York (1954)

22. Schwarz, H.A.: Gesammelte Mathematische Abhandlungen, vol. I. Springer, Berlin (1890) 